\title{
A case report on the legal assurance of ACP in collective culture
}

Hirotomo Miyatake ${ }^{1}$, Akihiko Ozaki ${ }^{2}$, Yasuhiro Kotera ${ }^{3}$, Ryo Sakamoto ${ }^{4}$, Divya Bhandari ${ }^{5}$, $\mathrm{Yu} \mathrm{Uneno}^{6}$, and Hiroyuki Beniya ${ }^{1}$

${ }^{1}$ Orange Home-Care Clinic

${ }^{2}$ Joban Hospital

${ }^{3}$ University of Derby

${ }^{4}$ Visina Home care

${ }^{5}$ Medical Governance Research Institute

${ }^{6}$ Kyoto University Graduate School of Medicine Faculty of Medicine

October 4, 2021

\begin{abstract}
This case report presents one important aspect of promoting ACP in Japanese culture, which is different from Western cultures. The report suggests the possibility that legal support contributes to the promotion of ACP, while considering differences in cultural contexts.
\end{abstract}

\section{A case report on the legal assurance of ACP in collective culture}

Hirotomo Miyatake ${ }^{1}$, MD; Akihiko Ozaki ${ }^{2}, \mathrm{MD}, \mathrm{PhD}$; Yasuhiro Kotera ${ }^{3}$, PhD; Ryo Sakamoto ${ }^{4}$, Divya Bhandari $^{5}$, Yu Uneno ${ }^{6}$, MD; Hiroyuki Beniya ${ }^{1}$, MD.

${ }^{1}$ Orange Home-Care Clinic, Fukui, Japan

${ }^{2}$ Department of Breast Surgery, Jyoban Hospital of Tokiwa Foundation, Iwaki, Japan

${ }^{3}$ College of Health, Psychology and Social Care, University of Derby, Derby, United Kingdom

${ }^{4}$ Visina Home care, Tokyo, Japan

${ }^{5}$ Medical Governance Research Institute, Tokyo, Japan

${ }^{6}$ Department of Therapeutic Oncology, Graduate School of Medicine, Kyoto University, Kyoto, Japan

*Corresponding author:

Hirotomo Miyatake, M.D.

Orange Home-Care Clinic, Fukui City, Fukui 910-0018, Japan

Phone: +81-776-21-3333, Fax: +81-776-21-3353

E-mail: hmiyatake@orangeclinic.jp

\section{Abstract}

This case report presents one important aspect of promoting ACP in Japanese culture, which is different from Western cultures. The report suggests the possibility that legal support contributes to the promotion of ACP, while considering differences in cultural contexts. 


\section{Key Clinical Message}

Legal support for ACP may be important to promote the practice of ACP in Japan. Legal support does not necessarily mean that ACP will always yield positive outcomes, however it can support preserving the foundation of ACP, respecting the individual's wish in relationship-conscious decision-making culture.

Key words: home care, ACP, culture, legal assurance

Word Count: 2161 words

\section{Introduction}

With the surge in a number of the aging population globally, the importance of prevention and appropriate management of chronic diseases is increasing. As a result, choices of treatments are becoming more complex and difficult for patients to understand. Moreover, supporting patients in making various decisions, including treatments choices, is challenging particularly, during their end-of-life decision as it involves various ethical issues.

In recent years, Advance Care Planning (ACP) has become a popular choice as it enables patients and their families to make a timely decision about future treatment options. In the $1960 \mathrm{~s}$, there was a wave of criticism of medical paternalism in the United States and movements to clarify living wills and to make Advance Directive (AD). Accordingly, in 1991, the Patient Self-Determination Act was enacted in the United States, requiring hospitals to provide information about AD and to inform patients that they had the right to receive or refuse treatment. Although AD was considered suitable for implementing patient intentions, change in the patient's decision over time caused problems in executing such fluctuating and unclear AD records. Therefore, the ACP that emphasizes reaching conclusions rather than merely recording them, was advocated. Recent studies have shown that ACP improves the quality of end-of-life care and satisfaction of patients and families, reduces stress, anxiety, depression in bereaved families ${ }^{1}$, and decreases the frequency of hospitalizations ${ }^{2}$, highlighting its efficiency and effectiveness in supporting patients decision.

In Japan, studies on the decision-making process for medical treatment and care in the end-of-life had been conducted since 1987, and the incident of a ventilator removal for euthanasia in the Imizu municipal hospital in 2006 led to rapid progress on the decision-making for medical treatment and care in the end-of-life in recent years. "Guideline for the Decision-Making Process for Terminal Care" was formulated in 2007, and it was revised as "Guideline for the Decision-Making Process for Medical treatment in the end-of-life" in 2018. In this revision, the importance of ACP approach was further emphasized as "individual's wishes can change in accordance with changes in physical and psychological conditions, policy of medical treatment and care, and his/her desire to live, etc., which need to be discussed on a daily basis." ${ }^{3}$ Furthermore, the Ministry of Health, Labor and Welfare (MHLW) commissioned the "Project for Improving the Medical System in the End-of-life, and Education for Implementing End-of-Life Discussion" (E-FIELD), which is an educational project for medical professionals to promote ACP, has been conducted nationwide to further promote and implement ACP in the medical field ${ }^{3}$. However, ACP was originally developed in the United States and Europe with proper legal support. Implementing ACP in Japan without such legal backing can create multiple issues that need to be explored. We are presenting a case study that demonstrates that the lack of strict legal guidance can cause a problem for the medical team to follow the patient's desire at the end-of-life care.

\section{Case}

A patient aged 97-year-old man living with his eldest daughter and her family used to regularly visit a general hospital for hypertension and chronic kidney disease. In January 2016, he developed urinary incontinence, frequent urination, and constipation, which he had never experienced before, and visited the emergency department of the general hospital. He was treated with sennoside tablet $12 \mathrm{mg}$ for constipation, however, his symptoms did not ease until three days after administrating medicine. So, at the request of his elder daughter, the attending physician visited the patient and prescribed two tablets of magnesium oxide 330mg in morning and evening to control defecation. While patients experienced relief from constipation and urinary 
incontinence, his urinary frequency was even prolonged. As blood test showed a high prostate specific antigen (PSA) level of $139.0 \mathrm{ng} / \mathrm{mL}$, the patient and his eldest daughter were informed about the possibility of prostate cancer. Considering the patient's age, he and his eldest daughter denied to undergo a thorough examination and choose to monitor the patient's condition with regular blood tests. In addition, the patient began using home care nursing facilities to follow up with his families and to prevent his Activities of Daily Living (ADL) from deteriorating.

With the help of his eldest daughter, he started keeping records of his urination frequency and defecation status and managed to take care of his health. He used to express gratitude to his eldest daughter who was his primary caregiver. However, sometimes he expressed a rather entitled attitude, commenting "it is expected for children to take care of their parents". Furthermore, he denied using day-care or short stay services, despite multiple recommendations from his daughter, doctors, and support specialists. His ADL began to decline prominently, and the frequency of urinary incontinence was increased. As the daughter was forced to deal with the patient's urination, she was tried over time and had difficulty accepting changes in her father's condition caused by senility. In January 2019, we received an emergency call explaining that the patient suddenly experienced strong back pain and was unconscious. When the doctor visited the patient's residence, he regained his conscious back. The patient and family members were explained about the possibility of a sudden change in his condition, but the patient assured the doctor that there was no need for him to go to the hospital. Although the family wanted to fulfill the patient's desire, they were worried about continuing the medical treatment at home and were particularly concerned about managing such emergencies.

His daughter felt strongly that she needed to care for him, which made it difficult for her to balance household chores and caregiving. So, with approval from the patient, they started using day-care and short-stay services from February 2019. There were some instances where the daughter also had difficulty accepting the fact that her father was aging with cognitive and physical decline, so she ended up reprimanding him for his behavior. Furthermore, she was worried as he refused to get admission to the hospital even in the emergency. To come up with the best possible solution, the home care team, which consisted of medical staff and care managers, discussed with the patient and his family about his future medical treatment plan. However, as the patient was adamant about his desire to continue home treatment, it was difficult to come up with a final solution that could address the desire of patient and at the same time reduce the burden of care on his family.

In June 2020, we received an emergency call with the complaint of a fever of 37.8 and increase of sputum production after sobbing at breakfast. At the time of the visit, his physical examination were temperature 38.2 , blood pressure $162 / 93 \mathrm{mmHg}$, pulse 90 / min, a saturation of percutaneous oxygen $89 \%$ at room air, respiration rate $26 / \mathrm{min}$ and also the presence of lung murmur. So, aspiration pneumonia was suspected. If physicians had to follow the patient's wishes at that stage, medical staff would have considered introducing home oxygen and daily antibiotic infusion by home care nurses to treat him at home. However, considering the family's mental state and their inability to care for a patient at home, physicians made a comprehensive judgment to take the patient to the hospital for emergency care. After that, he was transferred to a recovery hospital for rehabilitation. About a month later, the family informed that he had been admitted to a nursing home affiliated with the hospital and there was no further need for a home visit.

\section{Discussion}

The present case shows the difficulty that health care providers experience in serving as advocates for their patients in the absence of robust legal backing.

In the United States, the Patient Self-Determination Act was enacted in 1991. Early discussions about ACP have shown to enhance care that is consistent with the patient's goal, leading to positive family outcome. ${ }^{4}$ Advance Directive, developed based on the results of discussions, have legal force. ${ }^{5}$ In Europe, legislation on patient self-determination has led to active discussions and increases satisfaction with care. ${ }^{6}$ Euthanasia legislation in the Netherlands guarantees patients' rights, considering the importance of the discussion. It also recognizes the right of the physician to refuse to be involved when patients request euthanasia. In 
Canada, MAiD(Medical Assistance in Dying) has been legislated, which has led to more discussion about self-determination. ${ }^{7}$ However, self-determination is only recommended in the medical guidelines and there is no legal framework in Japan. In order to implement ACP, clear communication is essential, especially in home care. Regular patient visits provide more opportunities and time to talk to patients and their families, making it easier to advance ACP. In such a situation, having a legal ground for ACP may facilitate the discussion of respecting patients' rights, particularly in the context of Japan.

Many Japanese follow a cultural virtue of practicing "relationship-conscious self-decisions" because they prioritize family harmony and the result of which they want to align their personal decision with the will of the family or group to which they belong. Therefore, even if the patient's intentions differ from those of the family, the patient may try to harmonize by suppressing his or her own opinions. Also, as the patient becomes weaker and unable to share his/her opinion, family are more likely to make such decisions. In fact, most decision-makings in the dying stage are made between the physician and the family, and familycentered decision-making is widely practiced. ${ }^{8}$ In such cultural background, even if medical staff attempt to protect the patient's decision, it may cause conflicts between the patient and the family. In such conflicting situations, medical staff tend to proceed according to the wishes of the family to avoid trouble with the family members. This is one of the factors that can make it difficult to proceed with ACP in the Japanese contexts. In our case study, although the patient's desire was understood and was shared among the family and the medical staff, his cognitive function and ADL gradually declined and the family member opted for care according to their wish. The medical staff was also left with no choice but to follow the procedure according to the desire of family members. In general, it has been reported that the participation of caregivers in ACP promotes family acceptance regarding changing patient's condition and respect for patient's wishes, thereby facilitating end-of-life care at home. ${ }^{910}$ In Japan, it has been also reported that discussion of care goals between the doctor and the family can reduce conflicts in decision making. ${ }^{11}$ However, in the present case, although the caregivers discussed the issue, they were not able to participate in ACP, and it was difficult to make policy decisions in accordance with the patient's wishes. Given the cultural background of Japan, where it is difficult to follow/implement a patient's wishes, it is important to have a legal basis for the discussion so as not to deviate from the patient's wishes. On the other hand, there are some points that do not fit into the Japanese culture regarding the establishment of a legal basis for ACP. While some patients rely heavily on the opinions of their families, some patients leave all decisions to family members, even if they are competent to make their own decision. As a result, the patient may end up not understanding what is happening to them. In addition, there are cases where the patient and the family are unable to harmonize, resulting in a breakdown of the relationship and difficulty in decision making. However, it has been reported that decision-making conflicts are reduced when the surrogate decision-maker is aware of the patient's preferences, ${ }^{1213}$ and when the family fully understands the patient's wishes, the stress of decisionmaking can be reduced by achieving harmony. In such a cultural background, if the ACP has a legal basis and patients are half-heartedly encouraged to make decisions on his or her own, it will be difficult to agree with the whole process, which may result in high stress. In some cases, leaving decision-making to the family rather than the patient may increase the patient's satisfaction, and over-emphasizing the patient's self-determination can result in a loss of direction. Therefore, careful judgment and evaluation should be done before proceeding with any decision.

It has been suggested that cooperation of ACP between patients and their families may improve decisionmaking conflicts, ${ }^{14}$ and it would be meaningful for patients and their families to actively promote ACP in-home care. However, it would be difficult to implement effective ACP because patients' rights can easily become ambiguous in collective cultures such as Japan. Although legal guarantees such as the Dutch Euthanasia Act and Maid of Canada may not always work. However, a legal rationale that guarantees the rights of both patients and providers in $\mathrm{ACP}$ and respects the goals and values of the patient ${ }^{15}$ may contribute to promoting discussion of respect for the wishes of the patient in a collective culture that tends to favor the wishes of the family. Moreover, if this is deemed difficult due to various political barriers, it may be necessary to consider how to promote patient-based ACP while being aware of the lack of such barriers.

\section{Acknowledgements}


The authors express their sincere gratitude to all the staff members involved in this case.

\section{Conflict of interest statement}

Dr. Ozaki received a personal fee from MNES INC. outside of the submitted work.

\section{Funding statement}

This study did not receive any funding.

\section{Ethical approval}

This research meets the ethical guidelines and adheres to Japan's local legal requirements. An ethical review is not required for this type of article.

\section{Consent of publication}

Written, informed consent was obtained from the patient's family for publication of this case report and any accompanying images.

\section{Author contributions}

HM, and AO: wrote the initial draft of the manuscript. HM, AO, YK, RS, DB, YU and HB: assisted in the preparation of the manuscript. HM is the lead author and responsible for submission. All authors critically reviewed and revised the manuscript. HM and HB had full access to the data, and controlled the decision to publish, and accept full responsibility for the work.

Reference

1. Jeong S, Barrett T, Ohr SO, et al. Study protocol to investigate the efficacy of normalisation of Advance Care Planning (ACP) for people with chronic diseases in acute and community settings: a quasi-experimental design. BMC Health Serv Res. 2019;19(1):286.

2. Brinkman-Stoppelenburg A, Rietjens JA, van der Heide A. The effects of advance care planning on end-of-life care: a systematic review.Palliat Med. 2014;28(8):1000-1025.

3. Cheng S-Y, Lin C-P, Chan HY-l, et al. Advance care planning in Asian culture. Japanese Journal of Clinical Oncology.2020;50(9):976-989.

4. Bernacki RE, Block SD. Communication about serious illness care goals: a review and synthesis of best practices. JAMA Intern Med.2014;174(12):1994-2003.

5. Vearrier L. Failure of the Current Advance Care Planning Paradigm: Advocating for a CommunicationsBased Approach. HEC Forum.2016;28(4):339-354.

6. Ten Koppel M, Pasman HRW, van der Steen JT, et al. Consensus on treatment for residents in long-term care facilities: perspectives from relatives and care staff in the PACE cross-sectional study in 6 European countries. BMC Palliat Care. 2019;18(1):73.

7. Patel T, Christy K, Grierson L, et al. Clinician responses to legal requests for hastened death: a systematic review and meta-synthesis of qualitative research. BMJ Support Palliat Care. 2021;11(1):59-67.

8. Kanoh A, Kizawa Y, Tsuneto S, Yokoya S. End-of-Life Care and Discussions in Japanese Geriatric Health Service Facilities: A Nationwide Survey of Managing Directors' Viewpoints. Am J Hosp Palliat Care. 2018;35(1):83-91.

9. Abadir PM, Finucane TE, McNabney MK. When doctors and daughters disagree: twenty-two days and two blinks of an eye. J Am Geriatr Soc. 2011;59(12):2337-2340.

10. Ratner E, Norlander L, McSteen K. Death at home following a targeted advance-care planning process at home: the kitchen table discussion.J Am Geriatr Soc. 2001;49(6):778-781. 
11. Tsuda S, Nakamura M, Miyachi J, et al. Decisional Conflict in Home Medical Care in a Family-Oriented Society: Family Members' Perspectives on Surrogate Decision Making from a Multicenter Cohort Study. $J$ Palliat Med. 2019;22(7):814-822.

12. Vig EK, Starks H, Taylor JS, Hopley EK, Fryer-Edwards K. Surviving surrogate decision-making: what helps and hampers the experience of making medical decisions for others. J Gen Intern Med.2007;22(9):12741279 .

13. Braun UK, Beyth RJ, Ford ME, McCullough LB. Voices of African American, Caucasian, and Hispanic surrogates on the burdens of end-of-life decision making. J Gen Intern Med.2008;23(3):267-274.

14. Tay DL, Ellington L, Towsley GL, Supiano K, Berg CA. Evaluation of a Collaborative Advance Care Planning Intervention among Older Adult Home Health Patients and Their Caregivers. J Palliat Med.2020;23(9):1214-1222.

15. Hooper S, Sabatino CP, Sudore RL. Improving Medical-Legal Advance Care Planning. J Pain Symptom Manage. 2020;60(2):487-494. 